\section{Relational judgment of sound intensity by young children*}

\author{
ALLEN L. CARTER, KENNETH S. RICKER, and DAVID A. CORSINI† \\ University of Georgia, Athens, Georgia 30601
}

Developmental changes in the ability to make relational judgments of sound intensity were examined with 3-, 4-, and 5-year-old children. The hypothesis that correct judgments of "louder" precede correct judgments of "softer" was supported. The results were discussed with reference to the development of the understanding of logical relations as proposed by Piaget. The role of magnitude of perceptual information in the development of relational judgments also was discussed.

While field testing a science education unit on sound with firstand second-grade children, it was noted that the children more correctly chose the louder of two sounds than the softer of two sounds. Since the louder-softer relationship is logically reciprocal for adults, the tendency for more correct judgments of louder was at first thought to be artifactual. Inspection of the educational unit, however, showed that the sounds used in the unit were well above threshold and that the sounds used in each comparison were sufficiently distinct from one another. Pretraining was also conducted to insure that the children understood the verbal labels "louder" and "softer." The same differences mentioned above were maintained following training. Rather than being artifactual, the differential ability in using the two comparatives seemed to reflect the developing organization and utilization of perceptual information within a logical framework.

A review of the theoretical and experimental literature on relational comparisons with children showed that similar discrepancies have been noted for other dimensions. The development of the ability to deal with relational stimuli and to make comparative judgments with those stimuli has been discussed by Piaget (1952). Piaget states that at about the age of 4 , the child has the ability to make relational discriminations; but, curiously, it is not until about age 7 that the child can successfully seriate based on the same relation.

This finding has been replicated by Elkind (1964). Based on this evidence, Piaget (1968) asserts that until age 6 or 7 , the ability to understand

*This study was supported in part by the Office of General Research and the Research and Development Center in Educational Stimulation, University of Georgia. This study was reported as part of a Master's thesis by the senior author.

TReprint requests: D. Corsini, Psychology Department. University of Georgia, Athens, Georgia 30601 . relations is not incorporated as a logical process, but rather seems to exist as a percpetual process which is used intuitively. Until the age of 6 , the preoperational child performs a relational discrimination without benefit of the abstracted notion of a comparative relation or the realization that comparatives pertaining to a single dimension (larger-smaller, e.g.) are logical inverses. Thus, one might expect "larger" judgments at this age to be somewhat independent of "smaller" judgments of the same pair of stimuli. This has, in fact, been found in every investigation to date.

Thrum (1935) examined childrens' (aged 2:6 to $4: 0$ ) ability to select from a group of three tridimensional forms, The biggest object was correctly chosen by $88 \%$ of the Ss. Sixty-eight percent correctly chose the littlest object, and $48 \%$ correctly chose the middle-sized object. Beilin (1964, 1965 ) found that approximately $94 \%$ of kindergarten children could judge correctly whether $\mathbf{E}$ or $\mathbf{S}$ had more objects, whereas only $85 \%$ identified who had fewer objects, and $48 \%$ correctly indicated equality. Smedslund (1966), using children ranging in age from $5: 2$ to $7: 0$ in an experimental study of the logical operations of addition and subtraction, noted that there was a differential facility: "more" judgments (number of objects), as opposed to "less" judgments, were easier for the children.

The foregoing results indicate that not only are relational judgments somewhat independent, but they are consistent in a way related to magnitude regardless of dimension. In the order of difficulty, "A greater than $B$ " appears to be the easiest, "A less than B" is somewhat harder, and "A equal to $B$ " seems to be the most difficult for the preoperational child to master. This order of difficulty appears across the dimensions of area, size, and numerosity (Thrum, 1935; Beilin, 1964, 1965; Smedslund, 1966).

It is tempting to assert along with
Beilin (1964) that the order of difficulty appears because the child has had more practice with or exposure to the label identifying the "A is greater than B" relation. There is some evidence for this point of view: Braine (1965) notes that the word "more" is one of the earliest occurring pivotal words in the child's emerging vocabulary. It is not clear, however, from Braine's work if the word is used as a true comparative. "More milk," for example, would more likely mean "provide milk again" than "give me more than last time." In any case, the "A is greater than $B$," "A is less than $B$," and "A is equal to $B$ " order of difficulty first appeared in Thrum's study using the words "biggest" and "littlest." It was also evident in the study that led to this investigation, with the words "louder" and "softer." Even if the consistent order of difficulty is a result of differential familiarity with the terms involved, one must still ask, why is there more exposure and practice with the " $\mathrm{A}$ is greater than $B$ " relation than with the "A is less than B" relation? Is it because adults are raised in the same fashion? Or is it because the relations are dependent on perceptual operations which themselves make describing comparative situations easier in terms of "more," "larger," and "louder" than "less," "smaller," and "softer"?

All of the previous research on the ability of children to make relational judgments has involved visual presentation of stimuli. Since visual presentation is almost always simultaneous and of indeterminate duration while aural presentation is almost always successive and of fixed duration, one must be cautious in generalizing the results from the visual to the aural mode. The present study systematically investigates developmental changes in the ability to make louder-softer judgments; first, it seeks to determine if auditory judgments follow the pattern established for visual comparisons and, second, to determine how several variables affect comparative judgments of magnitude by young children. From previous research with visual stimuli and the findings in field testing the science unit, it was expected that "louder" judgments would be made more correctly than would "softer" judgments and that the ability to deal with these concepts would increase with age. The influence of several other variables was not predicted but was included in an attempt to better understand this phenomenon.

\section{METHOD Design}

The problem was investigated using 


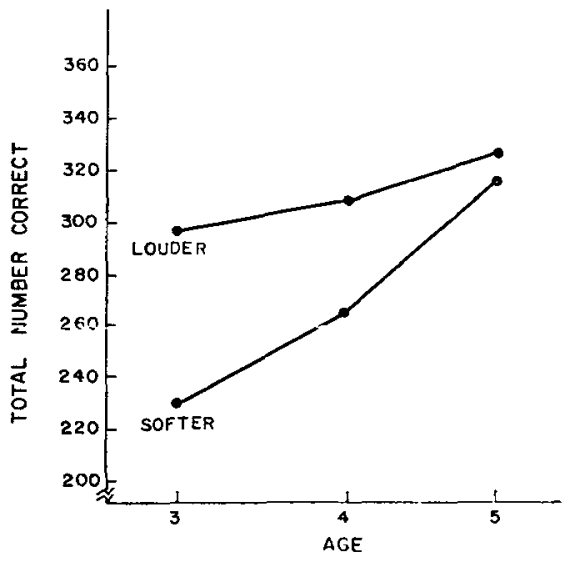

Fig. 1. Interaction between age and type of comparison.

a nested design involving age, comparison type, presentation order, and intensity level. An explanation of these variables follows.

Age level. As indicated previously, Piaget's preoperational stage, ages 4-6, appears to be the critical period for development of the ability to accurately make relational or comparative judgments about stimuli which differ along some dimension. This investigation, then, was to have included 4-, 5-, and 6-year-olds. The 6-year-olds, however, performed above the discriminative power of the measure. Their performance was essentially perfect. This was probably a result of their experiences in an experimental school which was the source of Ss for this study. Therefore, 3-, 4-, and 5-year-olds were used as Ss.

Comparison type. This variable refers to the comparative term used (louder-softer) and was intended to reveal whether or not accuracy was a simple function of the specific choice required. It was expected, on the basis of previous observation and the literature on relational judgment for visual dimensions, that children would be able to respond with more consistent accuracy when asked to choose the louder of two sounds than when asked to choose the softer of two sounds.

Presentation order. A pair of sounds may be presented in two different temporal orders: more intense-less intense and less intense-more intense. There is some evidence to indicate that children at this age may develop temporal position habits, usually choosing the last alternative (most recent). Sigel and Goldstein (1969) showed, along with Doehring (1969), that temporal position controls are necessary for "choice" type experiments with young children. Presentation order was included as an experimental variable, however, in order to determine if it would interact with the comparative type. It was felt that there might be an optimal presentation order for a particular comparison. For example, "A greater than B" comparisons might be facilitated if the louder sound appears first in the pair or vice versa.

Intensity levels. Rather than using one pair of sounds for comparison or randomizing the intensity levels, three pairs of sounds were chosen: one from the higher end of the intensity dimension, one from the lower end, and one which spanned the dimension. The upper and lower pairs were equated in terms of the intensity differences between the sounds in each pair. The middle pair contained one sound from the more intense range and one from the less intense range such that the intensity difference was twice the differences between the other two pairs. This arrangement was devised in order to assess the possible effects of absolute intensity differences on relative judgments.

\section{Subjects}

The Ss were 60 children enrolled in the Field Center in Clayton County, Georgia, an adjunct of the Research and Development Center for Early Educational Stimulation at the University of Georgia. Twenty of the children were 3-year-olds, 20 were 4-year-olds, and 20 were 5-year-olds. The children were chosen randomly from their respective age groups at the school, with the restriction that children who failed or passed with some question a standard hearing test administered by the school were excluded from participation in the study. Three Ss were replaced because of fear of the $E$ or apparatus. To avoid a differential sex effect, 10 of the $S$ s in each age group were male and 10 were female,

All of the children had been exposed to the words "louder" and "softer" by a music teacher who taught the children. These children began their schooling at age 3 and probably are not representative of the normal population of $3-, 4-$, and 5-year-olds. The majority of the children appeared to have come from a middle class socioeconomic environment. These Ss had participated previously in other experimental studies and seemed to have a task-oriented attitude.

\section{Apparatus}

The apparatus was a Sony TC-630 tape recorder, the speakers for which were used to present pairs of atonal sounds for comparison and judgment by the Ss. The sounds were previously recorded onto Sony 1/4-in. magnetic

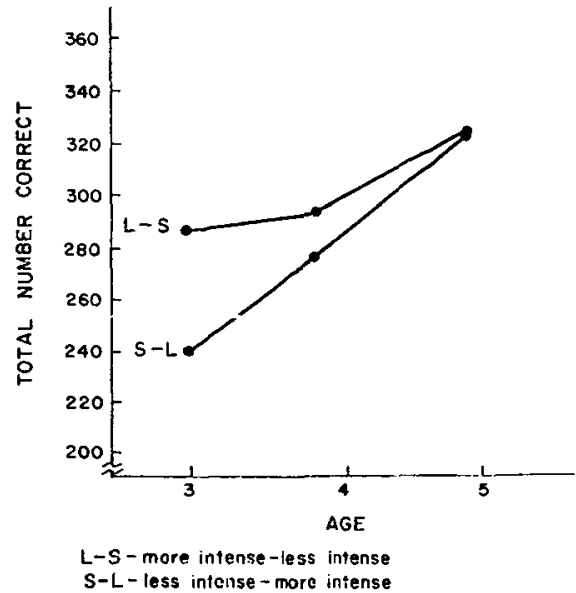

Fig. 2. Interaction between age and order of sound presentation.

tape. The recorder was calibrated to produce each sound at a predetermined intensity level at a distance of $36 \mathrm{in}$. from the speakers. The sounds were checked with a General Radio Sound Level Meter and were found to be accurate to .5 of $1 \mathrm{~dB}$.

\section{Stimuli and}

Stimulus Arrangement

The stimuli were sounds of atonal quality quite similar to "white noise." The sounds had a duration of $2 \mathrm{sec}$ and a separation of $1 \mathrm{sec}$ between sounds in a given comparison pair. A ready signal preceded each pair by 5 sec. Three comparison pairs were chosen. One pair of sounds was chosen from the lower end of the intensity dimension, one pair was chosen from the upper end of the intensity dimension, and one pair was chosen which contained one sound from the lower and one sound from the upper ends of the intensity dimension.

In order to ensure that the performance in judging would be comparable between the lower and upper pairs in terms of the perceived intensity difference between the sounds in the lower pair and the perceived intensity difference between the sounds in the upper pair, Stevens and Davis's (1938) adaptation of Riesz's (1933) work on difference limens was used to equate the upper pair with the lower pair in terms of DLs.

$P_{1}$, the lower pair, was composed of one sound at $40 \mathrm{~dB}$ and one sound at $59 \mathrm{~dB}$. The number of discriminable steps in this interval is $40 . P_{3}$, the upper pair, was composed of one sound at $70 \mathrm{~dB}$ and the other at $82 \mathrm{~dB}$-again, an interval of $40 \mathrm{DLs}$. $P_{3}$, the third pair, was composed of one sound at $50 \mathrm{~dB}$ and one at $77 \mathrm{~dB}$, 
a difference of 80 DLs.

Each of the three pairs was presented once for a "louder" judgment by $S s$ and once for $a$ "softer" judgment. The position of the more intense sound within each pair (e.g., whether it preceded or followed the less intense sound) generated two orders of presentation for each pair. This design produced 12 distinct combinations of the variables: three sound levels, two comparisons, and two orders of presentation. In order to improve the reliability of the measure, each of the 12 combinations was presented three times, yielding 36 trials. All 12 combinations were presented before any one combination was repeated. Three random orders of the 12 combinations were used.

\section{Procedure}

The experiment was conducted in one of two rooms which were quiet and reasonably free of distraction. Each $S$ was taken individually from class and escorted by $E$ to the experimental setting. $E$ showed the apparatus to $S$, explaining that it was a device for making sounds. $S$ was told that he would hear two sounds, one from each speaker, and then would be asked to choose (by pointing) the one that made the louder (or softer) sound. All of the Ss indicated that they knew what "louder" and "softer" meant. If there was any hesitancy by $S$, he was asked to make a louder sound (by rapping the table) than the $E$, then a softer sound.

The 3-year-olds and some of the 4-year-old Ss paid more attention to the task if they were allowed to run from the chair, which was set in a predetermined position ( $3 \mathrm{ft}$ from the speakers), after presentation of the pair and touch the correct speaker. This provided more of a game-like atmosphere and made the situation less threatening.

The $E$ told the child both before and after each presentation which sound to choose (louder or softer). The procedure took $15-20 \mathrm{~min}$ for each child.

None of the Ss verbalized or gave nonverbal indications of difficulty in using locational cues to determine which speaker the sounds came from.

\section{RESULTS}

The data, the number of correct choices, were subjected to a 3 (age) by 2 (comparison type) by 2 (stimulus order) by 3 (intensity level) analysis of variance. All four main effects were highly significant (all ps $<.01$ ). In addition, age interacted significantly with each of the other three variables. No other interactions were significant. Since each main effect was qualified by an age interaction, only the latter will be discussed.

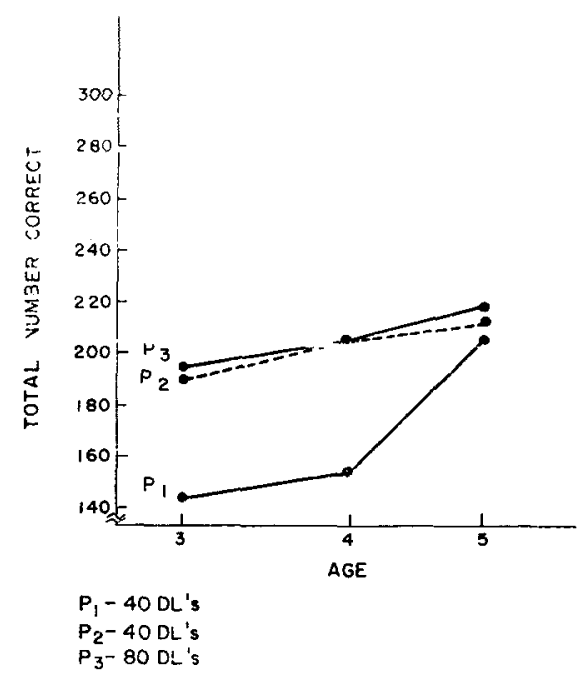

Fig. 3. Interaction between age and intensity level of sound pair.

\section{Age by Comparison}

The Age by Comparison, $F(2,57)=9.20, p<.01$, interaction is presented in Fig. 1. A Newman-Keuls analysis applied to the interaction showed that the difference in accuracy between choosing the louder and choosing the softer was significant for the 3-and 4-year-olds, but not for the 5-year-olds. It also revealed that the increase in accuracy with increasing age was due to improved accuracy in choosing the softer sound. The "louder", judgments were fairly accurate for all three age groups (no significant change), whereas each successive older age group was significantly more accurate in choosing the softer sound.

\section{Age by Order}

The Age by Order, $F(2,57)=3.50$, $p<.05$, interaction is presented in Fig. 2. A Newman-Keuls analysis demonstrated that the difference between orders of presentation was significant only for the 3-year-olds. In this case, the more intense-less intense order produced greater accuracy. Both orders showed progressive improvement with age. For the more intense-less intense order the improvement between 3 and 4 years was significant at the $p<.01$ level. Progressive improvement was also evident for the less intense-more intense order. Changes in accuracy from 3 to 4,4 to 5 , and 3 to 5 years were all found to be significant at the $p<.01$ level.

The Age by Intensity $F(4,114)=4.95, p<.01$, interaction is presented in Fig. 3. Performance with the sound pair from the more intense end of the scale $\left(P_{2}\right)$ and with the pair which contained sounds from both ends of the scale $\left(P_{3}\right)$ was relatively high across all three ages. It was the pattern of performance with the less intense sound pair which accounts for the significant main effect and interaction with age. The performance of both the 3 , and 4-year-olds was significantly poorer with the less intense sound pair than with the other two sound pairs $(p<.01)$. The performance of the 3 and 4-year-olds with the less intense pair was also significantly poorer than the performance of the 5-year-olds $(p<.01)$. The 5-year-olds performed equally well with the three sound pairs. There were no significant differences in the performance of the 3- and 4-year-old Ss in respect to intensity pairs.

\section{DISCUSSION}

The data on the 3-and 4-year-olds fell into the same pattern found previously by other investigators for preoperational children. The " $A$ is less than B" relation (softer) was more difficult for the $\mathrm{Ss}$ than the "A is greater than B" relation (louder). This finding thus adds the auditory intensity dimension to the list of visual dimensions (cubic size, area, and numerosity) for which differential accuracy in using reversible comparatives has been found. The 5-year-olds in this study did not differ significantly on any of the variables assessed. This was probably due to the special educational situation at the experimental school from which the Ss were drawn. The 5-year-olds had been in school for $2 \frac{1}{2}$ years.

Age level interacted with each of the other variables. There was a general improvement in accuracy with increasing age. The net effect of the improvement was to reduce the discrepancies in performance. There were no significant differences for 5-year-olds; the largest differences were obtained for 3-year-olds. This suggests that it is during this period of development-the preoperational stage-that the comparative relations lose some of their perceptual-intuitive character and begin to acquire logical properties.

An increased understanding of this phenomenon, or a hypothesis for further testing, is possible by analyzing the requirements for successful performance under the different conditions and the observations of the Ss" performance under these conditions.

The successive nature of the sound stimuli represents a state of affairs different from that of the usual simultaneous presentation used in visual studies. With successive presentation, the child is more or less forced to use the comparison order 
that the $\mathrm{E}$ has devised. The 3-year-olds had significantly more difficulty in responding to either louder or softer when the less intense sound was presented first, followed by the more intense. In addition, 3-year-olds performed more poorly when both sounds were relatively soft, regardless of the comparative used. These two bits of evidence suggest that the younger Ss were using the louder sound as a kind of perceptual anchoring point from which comparisons were made. It was, in fact, observed that some of the children pointed, during the presentation, at the speaker which emitted the louder sound. If the second sound was louder, they shifted to it regardless of the comparative asked for. After hearing both sounds and holding the louder with a point, they then decided which was correct. In the less intense-more intense order, the anchor could not be finally established until the second stimulus was heard. The necessity to shift anchor probably resulted in more error than in the order more intense-less intense where a shift was not required.

The child's difficulty was compounded when two relatively soft sounds were used $\left(P_{1}\right)$. Since the other two pairs $\left(P_{2}\right.$ and $\left.P_{3}\right)$, contained relatively intense sounds, the child might therefore have expected a loud sound to follow the soft sound which was heard first. When, instead, another soft sound was heard, the child became confused, having no anchor point at all. For those children who pointed to mark their place, the sequence seemed to go like this: the child listened to the first sound then pointed toward the other speaker, anticipating a louder stimulus. When the second sound was also relatively soft, the child wavered back and forth looking confused.

These observations suggest that "natural" comparisons which happen outside the laboratory are dependent on the attentional power of stimuli. That is, when two events occur in the perceptual field that share a common dimension, the source of stronger stimulation elicits a stronger orienting response and thereby becomes a natural fixation point from which comparison proceeds.

Relational thinking in preoperational children has not concerned many investigators, but the studies found all cortain a common theme which is represented in this study also: dimensions which are easily characterized as differing in magnitude, such as number, space, length, and sound intensity (stimuli that differ quantitatively rather than qualitatively), provide different tasks, depending on how the relationship between any two stimuli in the dimension is expressed. The task of expressing the relation " $\mathrm{A}$ is greater than B" seems to be easier for a child than the task of expressing the relation "A is less than B." Previous investigators have pointed out this difference for individual dimensions, but, before, no one had extracted the order of difficulty or magnitude phenomenon across dimensions and modalities. The addition of these results to prior findings adds weight to the conclusion that the magnitude phenomenon does not represent some experimental artifact, but rather appears to be a genuine property of verbal-relational thinking in the preoperational child.

The experimental question here can lead to other questions and implications which have a broader scope. It is felt that the child's experiences with the primary dimensions (i.e., those involving direct sensory experience of magnitude) form the basis for an abstract scale upon which can be superimposed abstract terms for magnitude comparison. Thus, the adult possesses an imaginary scale of magnitude whose units are in terms of distance and whose operations are in terms of direction. He can order objects on this scale according to whatever dimension he chooses-hardness, roundness, legality, etc. This magnitudinal ordering or comparing operation is usually considered to be a logical tool for cognitive organization when such abstract terms are manipulated. However, notice the close connection between the logical tool which is used by the operational child and its "perceptual intuitive" counterpart in the preoperational child. The development of the ability to manipulate logical relationships must have its beginning in the ability to manipulate more basic perceptual relationships.

The authors feel that it is this ground that, once broken, will yield the basic data on important questions now being pursued at higher age levels. The conservation tasks, for example, seem intimately related to the development of relational concepts. How these concepts are maintained under perceptual transformation is a question that must eventually involve study at lower age levels to determine how these concepts are formed and how they are utilized by the child.

The magnitude phenomenon has educational implications which should not be overlooked. For the kindergarten child or the first grader, instruction dealing with dimensional comparatives may be assimilated differently from what is conventionally supposed. The adult teacher who already has the abstract logical system for dealing with comparative relations can easily forget or be unaware that the child is in the process of building the system and that, therefore, the child may not comprehend explanations which assume he already has an abstracted notion of reversible comparatives. Teachers should be sensitive, therefore, to the difficulties that the child may have when provided with examples of the "A less than B" relation. In addition, for particular dimensions, practice should cut across the entire range of possible stimuli so as to aid the child in realizing that comparatives depend on relations and not absolutes. Finally, teachers should ensure that the child learns to respond on a purely relational basis by not confounding the relation with temporal presentation orders or other characteristics of the dimension involved.

\section{REFERENCES}

BEILIN, H, Perceptual-cognitive conflict in the development of an invariant area concept. Journal of Experimental Child Psychology, 1964, 1. 208-226.

BEILIN, H. Learning and operational convergence in logical thought development. Journal of Experimental Child Psychology, 1965, 2, 317-339.

BRAINE, M. D. S. The ontogeny of English phrase structure: The first phase. In $R$. A Anderson and D. P. Ausabel (Eds.), Readings in the psychology of cognition. New York: Holt, Rinehart, \& Winston, 1965. Pp. 303-320.

DOEHRING, D. H. Temporal sequence effects in auditory oddity discrimination. Perception \& Psychophysics, 1969, 6, 65-68.

ELKIND, D. Discrimination, seriation, and numeration of size and dimensional differences in young children: Piaget replication study VI. Journal of Genetic Psychology, 1964, 104, 275-296.

PIAGET, J. The child's conception of number. New York: Humanities Press, 1952 .

PIAGET, J. Six psychological studies. New York: Random House, 1968. Pp. 48-51.

RIESZ, R. R. The relationship between loudness and the minimum perceptible increment of intensity. Journal of the Acoustical Society of America, 1933, 4, 211-216.

SIGEL L S. \& GOLDSTEIN, A. G. Conservation of number in young children: Recency versus relational response strategies. Developmental Psychology, 1969, 1, 129-130.

SMEDSLUND, J, Microanaly sis of concrete reasoning, I. The difficulty of some combinations of addition and subtraction of one unit. Scandinavian Journal of Psychology, 1966, 7, 145-167.

STEVENS, S. S., \& DAVIS, H. Hearing: Its psychology and physiology. New York: Wiley, 1938 .

THRUM, $M$. E. The development of concepts of magnitude. Child Development, 1935, 6, 120-140.

(Received for publication March 11, 1971.) 